\title{
Correction to: Endoplasmic Reticulum Stress Plays a Key Role in Rotenone-Induced Apoptotic Death of Neurons
}

\author{
Poonam Goswami ${ }^{1,2} \cdot$ Sonam Gupta ${ }^{1} \cdot$ Joyshree Biswas ${ }^{1} \cdot$ Neeraj Joshi $^{3,4} \cdot$ Supriya Swarnkar $^{5} \cdot$ Chandishwar Nath $^{1}$. \\ Sarika Singh ${ }^{1,2}$
}

Published online: 22 June 2019

(C) Springer Science+Business Media, LLC, part of Springer Nature 2019

\section{Correction to: Mol Neurobiol (2016) 53:285-298}

https://doi.org/10.1007/s12035-014-9001-5

The original version of this article unfortunately contained a mistake.

The authors observed inadvertent error in Fig. $4 \mathrm{c}$ and wish to correct it. In this figure, the two images of $4 \mathrm{c}, \mathrm{Cont}+\mathrm{Sal}$ and Rot $0.1 \mu \mathrm{M}+\mathrm{Sal}$, have been wrongly presented.

The authors hereby publish the correct Fig. 4.

The online version of the original article can be found at https://doi.org/ $10.1007 / \mathrm{s} 12035-014-9001-5$

Sarika Singh

sarika_singh@cdri.res.in; ssj3010@gmail.com

1 Toxicology Division, CSIR- Central Drug Research Institute,

Lucknow, Uttar Pradesh 226031, India

2 Academy of Scientific \& Innovative Research (AcSIR), New

Delhi, India

3 Center for Gene Regulation in Health and Disease, Department of Biological Sciences, Cleveland State University, Cleveland, OH, USA

4 Department of Genetics, Harvard Medical School, Boston, MA, USA

5 Scripps Research Institute, Jupiter, FL, USA 


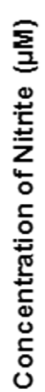

(a) 30$]$ Rotenone
- Rotenone+Salubrinal

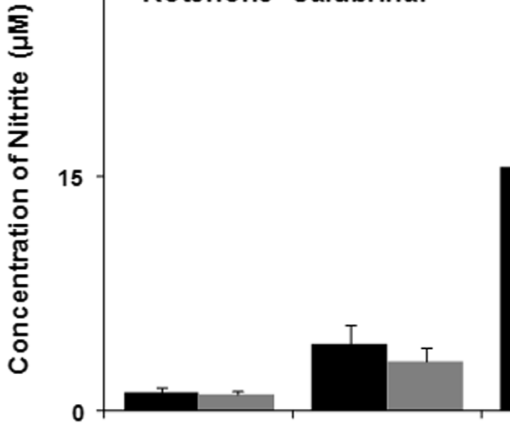

Control

$0.1 \mu \mathrm{M}$

\begin{abstract}
$0.5 \mu \mathrm{M}$
\end{abstract}

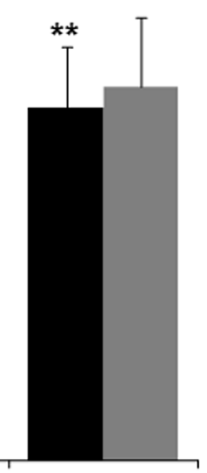

$1 \mu \mathrm{M}$

(b)

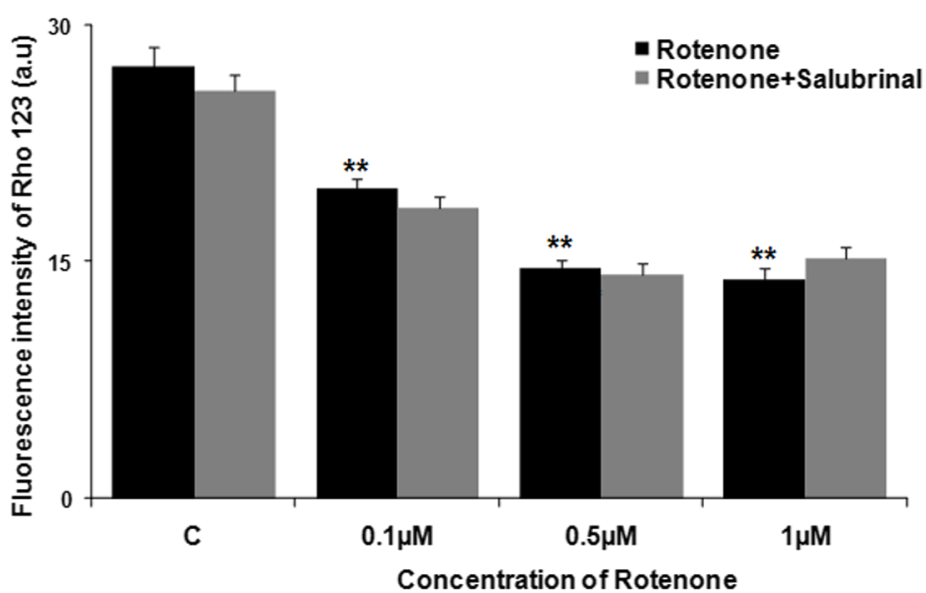

(c)

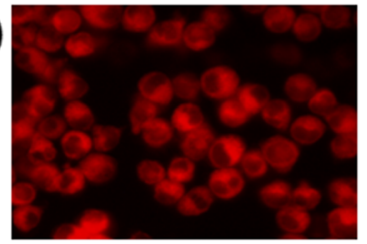

Control

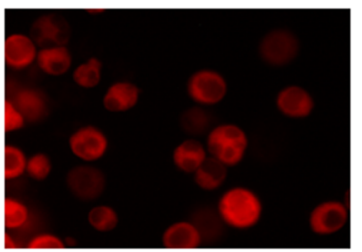

Rot $0.1 \mu \mathrm{M}$

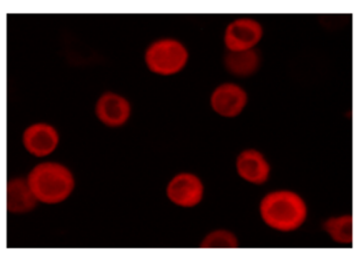

Rot $0.5 \mu \mathrm{M}$

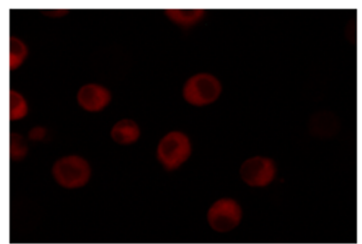

Rot $1 \mu \mathrm{M}$

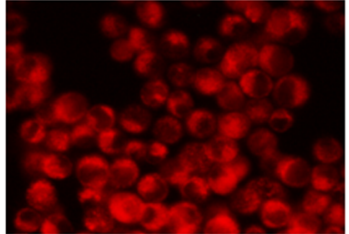

Control+Sal

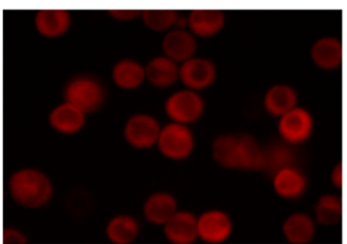

Rot $0.1 \mu \mathrm{M}+\mathrm{Sal}$

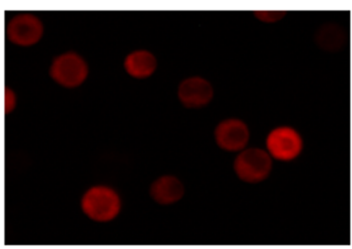

Rot $0.5 \mu \mathrm{M}+\mathrm{Sal}$

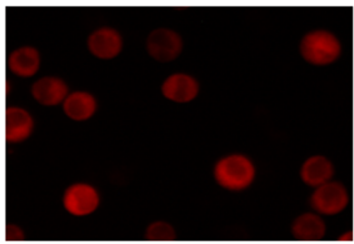

Rot $1 \mu \mathrm{M}+\mathrm{Sal}$
Fig. 4 a Graphical representation of nitrite levels after $16 \mathrm{~h}$ in rotenoneand rotenone + salubrinal $(25 \mu \mathrm{M})$-treated neurons. b Rhodamine 123 fluorescence depicting mitochondrial membrane potential after $16 \mathrm{~h}$ in rotenone- and rotenone + salubrinal $(25 \mu \mathrm{M})$-treated neurons. $\mathbf{c}$ Pictorial representation of fluorescent images of rhodamine 123 after rotenone

(Rot) and rotenone + salubrinal $($ Sal $25 \mu \mathrm{M})$ treatment. Data are expressed as mean \pm SEM, analyzed by ANOVA post hoc NewmanKeuls multiple comparison test. $* *=p<0.001$ (control vs. rotenone treated)

Publisher's Note Springer Nature remains neutral with regard to jurisdictional claims in published maps and institutional affiliations. 